\title{
Dissecting Aggregate Output and Labour Productivity Change
}

\author{
Bert M. Balk
}

\begin{tabular}{|l|l|}
\hline \multicolumn{2}{|l|}{ ERIM REPORT SERIES RESEARCH IN MANAGEMENT } \\
\hline ERIM Report Series reference number & ERS-2011-023-MKT \\
\hline Publication & November 2011 \\
\hline Number of pages & 22 \\
\hline Persistent paper URL & http://hdl.handle.net/1765/26893 \\
\hline Email address corresponding author & bbalk@rsm.nl \\
\hline Address & Erasmus Research Institute of Management (ERIM) \\
& RSM Erasmus University / Erasmus School of Economics \\
& Erasmus Universiteit Rotterdam \\
& P.O.Box 1738 \\
& 3000 DR Rotterdam, The Netherlands \\
& Phone: + 31104081182 \\
& Fax: $\quad+31104089640$ \\
& Email: info@erim.eur.nl \\
& Internet: $\quad$ www.erim.eur.nl \\
\hline
\end{tabular}

Bibliographic data and classifications of all the ERIM reports are also available on the ERIM website: www.erim.eur.nl 
ERASMUS RESEARCH INSTITUTE OF MANAGEMENT

REPORT SERIES

RESEARCH IN MANAGEMENT

\begin{tabular}{|c|c|}
\hline \multicolumn{2}{|c|}{ ABSTRACT AND KEYWORDS } \\
\hline Abstract & $\begin{array}{l}\text { This paper is about the relation between output and productivity measures for individual } \\
\text { production units and those for aggregates such as industries, sectors, or economies. In the } \\
\text { framework of discrete timeperiods several useful expressions are derived and confronted with } \\
\text { results from the literature. }\end{array}$ \\
\hline Free Keywords & Producer, profit, profitability, productivity, aggregation, index number theory \\
\hline Availability & $\begin{array}{l}\text { The ERIM Report Series is distributed through the following platforms: } \\
\text { Academic Repository at Erasmus University (DEAR), DEAR ERIM Series Portal } \\
\text { Social Science Research Network (SSRN), SSRN ERIM Series Webpage } \\
\text { Research Papers in Economics (REPEC), REPEC ERIM Series Webpage }\end{array}$ \\
\hline Classifications & $\begin{array}{l}\text { The electronic versions of the papers in the ERIM report Series contain bibliographic metadata } \\
\text { by the following classification systems: } \\
\text { Library of Congress Classification, (LCC) LCC Webpage } \\
\text { Journal of Economic Literature, (JEL), JEL Webpage } \\
\text { ACM Computing Classification System CCS Webpage } \\
\text { Inspec Classification scheme (ICS), ICS Webpage }\end{array}$ \\
\hline
\end{tabular}




\title{
Dissecting Aggregate Output and Labour Productivity Change
}

\author{
Bert M. Balk* \\ Rotterdam School of Management \\ Erasmus University \\ Rotterdam \\ E-mail bbalk@rsm.nl \\ Draft, October 31, 2011
}

\begin{abstract}
This paper is about the relation between output and productivity measures for individual production units and those for aggregates such as industries, sectors, or economies. In the framework of discrete timeperiods several useful expressions are derived and confronted with results from the literature.
\end{abstract}

Keywords: Producer; profit; profitability; productivity; aggregation; index number theory.

JEL code: C43, O47.

*Thanks to Giannis Karagiannis for some constructive comments on an earlier version. 


\section{Introduction}

Aggregate output or productivity growth or decline is the outcome of numerous actions at the level of individual production units. To guide industry policy it is insufficient to relay only on statistics of aggregate behaviour, but it is important to be able to dissect those measures into contributions of lower level entities.

As a starting point we consider the method of Dufour, Tang and Wang (2006) (DTW) for computing industry contributions to aggregate output and labour productivity change. In this paper I first reconstruct their method by using the notational framework of Balk (2009), (2010a), (2010b). This reconstruction reveals a number of things. First, it appears that the DTW method is asymmetric, in the sense that the Laspeyres, forward-looking perspective is used. This calls for the development of a symmetric alternative. Second, the DTW method expresses aggregate output change as a weighted product or sum of the components of subaggregate output change rather then subaggregate output change itself. This calls for the development of an alternative that links aggregate output change, subaggregate output change, and the components thereof.

Next, I turn to DTW's decomposition of aggregate labour productivity change and develop a number of alternatives. Though structurally similar, these alternatives differ from each other in details. It will also be shown that these alternatives correspond to formulas put forward in the literature where the language of continuous time was used. It is well known that the mathematics of continuous time and infinitesimal changes tends to obfuscate interesting features which emerge when one uses the framework of discrete time periods, as in this paper.

\section{The decomposition of real value added change}

Consider an ensemble $\mathcal{K}$ of consolidated production units. Think of an economy consisting of industries, or an industry consisting of enterprises. For each unit and period the KLEMS-Y accounting identity in nominal values reads

$$
C_{K L(k)}^{k t}+C_{E M S}^{k t}+\Pi^{k t}=R^{k t}(k \in \mathcal{K}),
$$

where $C_{K L(k)}^{k t}$ denotes the primary input cost, $C_{E M S}^{k t}$ the intermediate input 
cost, $R^{k t}$ the revenue obtained on gross output ${ }^{1}$, and $\Pi^{k t}$ the profit (defined as remainder). Intermediate input cost (on energy commodities, materials, and business services) and revenue concern generally tradable items. The items in the capital and labour classes, however, are specific for each unit; which explains why there is an additional subscript.

The KL-VA accounting identity then reads

$$
C_{K L(k)}^{k t}+\Pi^{k t}=R^{k t}-C_{E M S}^{k t} \equiv V A^{k t}(k \in \mathcal{K}) .
$$

It is assumed that $V A^{k t}>0(k \in \mathcal{K})$. Adding-up the KLEMS-Y relations over all the units does not deliver the KLEMS-Y relation for the ensemble $\mathcal{K}$, considered as a consolidated production unit, because of deliveries between units. However, if for all the tradable commodities output prices are identical to input prices, then it appears that adding-up the KL-VA relations over all the units delivers the KL-VA relation for the ensemble:

$$
C_{K L(\mathcal{K})}^{\mathcal{K} t}+\Pi^{\mathcal{K} t}=V A^{\mathcal{K} t},
$$

where $C_{K L(\mathcal{K})}^{\mathcal{K} t}=\sum_{k \in \mathcal{K}} C_{K L(k)}^{k t}, \Pi^{\mathcal{K} t}=\sum_{k \in \mathcal{K}} \Pi^{k t}$, and $V A^{\mathcal{K} t}=\sum_{k \in \mathcal{K}} V A^{k t}$.

In the National Accounts it is usual to measure (aggregate) output as (aggregate) real value added, which is calculated as nominal value added deflated by an appropriate price index relative to some reference period $b$. Thus, formally,

$$
\begin{aligned}
\tilde{V A^{\mathcal{K}} t} & \equiv V A^{\mathcal{K} t} / P_{V A}^{\mathcal{K}}(t, b) \\
& =\sum_{k \in \mathcal{K}}\left(R^{k t}-C_{E M S}^{k t}\right) / P_{V A}^{\mathcal{K}}(t, b)
\end{aligned}
$$

Let there also be given price and quantity indices such that revenue ratios and intermediate cost ratios can be split into price and quantity components,

$$
\begin{aligned}
R^{k t} / R^{k b} & =P_{R}^{k}(t, b) Q_{R}^{k}(t, b) \\
C_{E M S}^{k t} / C_{E M S}^{k b} & =P_{E M S}^{k}(t, b) Q_{E M S}^{k}(t, b) .
\end{aligned}
$$

Real gross output is then defined by $\tilde{R}^{k t} \equiv R^{k b} Q_{R}^{k}(t, b)$, real intermediate input use by $\tilde{C}_{E M S}^{k t} \equiv C_{E M S}^{k b} Q_{E M S}^{k}(t, b)$, the relative gross output price index by $\tilde{P}_{R}^{k}(t, b) \equiv P_{R}^{k}(t, b) / P_{V A}^{\mathcal{K}}(t, b)$, and the relative intermediate input price

\footnotetext{
${ }^{1}$ It is assumed that the units operate on a market so that output prices are available.
} 
index by $\tilde{P}_{E M S}^{k}(t, b) \equiv P_{E M S}^{k}(t, b) / P_{V A}^{\mathcal{K}}(t, b)$. Substituting all these definitions into expression (4) it appears that aggregate output can be written as

$$
\tilde{V A^{\mathcal{K} t}}=\sum_{k \in \mathcal{K}}\left(\tilde{P}_{R}^{k}(t, b) \tilde{R}^{k t}-\tilde{P}_{E M S}^{k}(t, b) \tilde{C}_{E M S}^{k t}\right) .
$$

This is an important building block for what follows.

We now consider aggregate output change, going from an earlier period 0 (also called base period) to a later period 1 (also called comparison period). This is naturally measured by $\tilde{V A^{\mathcal{K} 1}}-\tilde{V A^{\mathcal{K} 0}}$, which can be written as

$$
\begin{aligned}
& \tilde{V A^{\mathcal{K} 1}}-\tilde{V A^{\mathcal{K} 0}}= \\
& \quad \sum_{k \in \mathcal{K}}\left(\tilde{P}_{R}^{k}(1, b) \tilde{R}^{k 1}-\tilde{P}_{R}^{k}(0, b) \tilde{R}^{k 0}\right) \\
& \quad-\sum_{k \in \mathcal{K}}\left(\tilde{P}_{E M S}^{k}(1, b) \tilde{C}_{E M S}^{k 1}-\tilde{P}_{E M S}^{k}(0, b) \tilde{C}_{E M S}^{k 0}\right) .
\end{aligned}
$$

The two parts at the right-hand side of this expression can be decomposed according to the Laspeyres, forward-looking perspective, yielding

$$
\begin{aligned}
& \tilde{V A}^{\mathcal{K} 1}-\tilde{V A^{\mathcal{K} 0}}= \\
& \sum_{k \in \mathcal{K}} \tilde{P}_{R}^{k}(0, b)\left(\tilde{R}^{k 1}-\tilde{R}^{k 0}\right) \\
& +\sum_{k \in \mathcal{K}} \tilde{R}^{k 0}\left(\tilde{P}_{R}^{k}(1, b)-\tilde{P}_{R}^{k}(0, b)\right) \\
& +\sum_{k \in \mathcal{K}}\left(\tilde{P}_{R}^{k}(1, b)-\tilde{P}_{R}^{k}(0, b)\right)\left(\tilde{R}^{k 1}-\tilde{R}^{k 0}\right) \\
& -\sum_{k \in \mathcal{K}} \tilde{P}_{E M S}^{k}(0, b)\left(\tilde{C}_{E M S}^{k 1}-\tilde{C}_{E M S}^{k 0}\right) \\
& -\sum_{k \in \mathcal{K}} \tilde{C}_{E M S}^{k 0}\left(\tilde{P}_{E M S}^{k}(1, b)-\tilde{P}_{E M S}^{k}(0, b)\right) \\
& -\sum_{k \in \mathcal{K}}\left(\tilde{P}_{E M S}^{k}(1, b)-\tilde{P}_{E M S}^{k}(0, b)\right)\left(\tilde{C}_{E M S}^{k 1}-\tilde{C}_{E M S}^{k 0}\right) .
\end{aligned}
$$

Switching to relative changes (forward-looking growth rates) delivers

$$
\frac{\tilde{V A^{\mathcal{K} 1}}-\tilde{V A^{\mathcal{K} 0}}}{\tilde{V A^{\mathcal{K} 0}}}=
$$




$$
\begin{aligned}
& \sum_{k \in \mathcal{K}} \frac{\tilde{P}_{R}^{k}(0, b) \tilde{R}^{k 0}}{\tilde{V A} \tilde{R}^{\mathcal{K} 0}} \frac{\tilde{R}^{k 1}-\tilde{R}^{k 0}}{\tilde{R}^{k 0}} \\
& +\sum_{k \in \mathcal{K}} \frac{\tilde{P}_{R}^{k}(0, b) \tilde{R}^{k 0}}{\tilde{V A} A^{K 0}} \frac{\tilde{P}_{R}^{k}(1, b)-\tilde{P}_{R}^{k}(0, b)}{\tilde{P}_{R}^{k}(0, b)} \\
& +\sum_{k \in \mathcal{K}} \frac{\tilde{P}_{R}^{k}(0, b) \tilde{R}^{k 0}}{\tilde{V A}} \frac{\tilde{P}_{R}^{k}(1, b)-\tilde{P}_{R}^{k}(0, b)}{\tilde{P}_{R}^{k}(0, b)} \frac{\tilde{R}^{k 1}-\tilde{R}^{k 0}}{\tilde{R}^{k 0}} \\
& -\sum_{k \in \mathcal{K}} \frac{\tilde{P}_{E M S}^{k}(0, b) \tilde{C}_{E M S}^{k 0}}{\tilde{V A} \tilde{C}_{E M S}^{k 1}-\tilde{C}_{E M S}^{k 0}} \\
& -\sum_{k \in \mathcal{K}} \frac{\tilde{P}_{E M S}^{k}(0, b) \tilde{C}_{E M S}^{k 0}}{\tilde{V A} A_{E M S}^{K 0}} \frac{\tilde{P}_{E M S}^{k}(1, b)-\tilde{P}_{E M S}^{k}(0, b)}{\tilde{P}_{E M S}^{k}(0, b)} \\
& -\sum_{k \in \mathcal{K}} \frac{\tilde{P}_{E M S}^{k}(0, b) \tilde{C}_{E M S}^{k 0}}{\tilde{V A}} \frac{\tilde{P}_{E M S}^{k}(1, b)-\tilde{P}_{E M S}^{k}(0, b)}{\tilde{P}_{E M S}^{k}(0, b)} \frac{\tilde{C}_{E M S}^{k 1}-\tilde{C}_{E M S}^{k 0}}{\tilde{C}_{E M S}^{k 0}} .
\end{aligned}
$$

Notice that $\tilde{P}_{R}^{k}(0, b) \tilde{R}^{k 0} / \tilde{V A}{ }^{\mathcal{K} 0}=R^{k 0} / V A^{\mathcal{K} 0}$, which is the base period share of nominal revenue of unit $k$ in aggregate nominal value added, and that $\tilde{P}_{E M S}^{k}(0, b) \tilde{C}_{E M S}^{k 0} / \tilde{V A}{ }^{\mathcal{K} 0}=C_{E M S}^{k 0} / V A^{\mathcal{K} 0}$, which is the base period share of nominal intermediate input cost of unit $k$ in aggregate nominal value added. Hence, these shares are independent of the reference period $b$. This, however, does not hold for the growth rates, contrary to the assertion of DTW. Notice that, for instance,

$$
\frac{\tilde{R}^{k 1}-\tilde{R}^{k 0}}{\tilde{R}^{k 0}}=\frac{Q_{R}^{k}(1, b)-Q_{R}^{k}(0, b)}{Q_{R}^{k}(0, b)},
$$

which in general remains dependent on reference period $b$ data.

The right-hand side of expression (10) consists of six terms: the first and the fourth give the aggregate effect of quantity change, the second and the fifth give the aggregate effect of relative price change, and the third and the sixth give the aggregate effect of the interaction of quantity and price change. The entire expression corresponds to DTW's expression (2).

\section{Alternative decompositions}

Instead of decomposing aggregate output change according to the Laspeyresperspective, as in expression (9), one can use the Paasche, backward-looking 
perspective,

$$
\begin{aligned}
& \tilde{V A^{\mathcal{K} 1}}-\tilde{V A}{ }^{\mathcal{K} 0}= \\
& \quad \sum_{k \in \mathcal{K}} \tilde{P}_{R}^{k}(1, b)\left(\tilde{R}^{k 1}-\tilde{R}^{k 0}\right) \\
& \quad+\sum_{k \in \mathcal{K}} \tilde{R}^{k 1}\left(\tilde{P}_{R}^{k}(1, b)-\tilde{P}_{R}^{k}(0, b)\right) \\
& \quad-\sum_{k \in \mathcal{K}}\left(\tilde{P}_{R}^{k}(1, b)-\tilde{P}_{R}^{k}(0, b)\right)\left(\tilde{R}^{k 1}-\tilde{R}^{k 0}\right) \\
& \quad-\sum_{k \in \mathcal{K}} \tilde{P}_{E M S}^{k}(1, b)\left(\tilde{C}_{E M S}^{k 1}-\tilde{C}_{E M S}^{k 0}\right) \\
& \quad-\sum_{k \in \mathcal{K}} \tilde{C}_{E M S}^{k 1}\left(\tilde{P}_{E M S}^{k}(1, b)-\tilde{P}_{E M S}^{k}(0, b)\right) \\
& +\sum_{k \in \mathcal{K}}\left(\tilde{P}_{E M S}^{k}(1, b)-\tilde{P}_{E M S}^{k}(0, b)\right)\left(\tilde{C}_{E M S}^{k 1}-\tilde{C}_{E M S}^{k 0}\right) .
\end{aligned}
$$

Notice the minus sign on the interaction terms. Switching to backwardlooking relative changes delivers

$$
\begin{aligned}
& \frac{\tilde{V A^{\mathcal{K} 1}}-\tilde{V A^{\mathcal{K} 0}}}{\tilde{V A^{\mathcal{K} 1}}}= \\
& \sum_{k \in \mathcal{K}} \frac{\tilde{P}_{R}^{k}(1, b) \tilde{R}^{k 1}}{\tilde{V A} A^{\mathcal{K} 1}} \frac{\tilde{R}^{k 1}-\tilde{R}^{k 0}}{\tilde{R}^{k 1}} \\
& +\sum_{k \in \mathcal{K}} \frac{\tilde{P}_{R}^{k}(1, b) \tilde{R}^{k 1}}{\tilde{V A} A^{\mathcal{K} 1}} \frac{\tilde{P}_{R}^{k}(1, b)-\tilde{P}_{R}^{k}(0, b)}{\tilde{P}_{R}^{k}(1, b)} \\
& -\sum_{k \in \mathcal{K}} \frac{\tilde{P}_{R}^{k}(1, b) \tilde{R}^{k 1}}{\tilde{V A} A^{\mathcal{K} 1}} \frac{\tilde{P}_{R}^{k}(1, b)-\tilde{P}_{R}^{k}(0, b)}{\tilde{P}_{R}^{k}(1, b)} \frac{\tilde{R}^{k 1}-\tilde{R}^{k 0}}{\tilde{R}^{k 1}} \\
& -\sum_{k \in \mathcal{K}} \frac{\tilde{P}_{E M S}^{k}(1, b) \tilde{C}_{E M S}^{k 1}}{\tilde{V A}^{\mathcal{K} 1}} \frac{\tilde{C}_{E M S}^{k 1}-\tilde{C}_{E M S}^{k 0}}{\tilde{C}_{E M S}^{k 1}} \\
& -\sum_{k \in \mathcal{K}} \frac{\tilde{P}_{E M S}^{k}(1, b) \tilde{C}_{E M S}^{k 1}}{\tilde{V A}^{\mathcal{K} 1}} \frac{\tilde{P}_{E M S}^{k}(1, b)-\tilde{P}_{E M S}^{k}(0, b)}{\tilde{P}_{E M S}^{k}(1, b)} \\
& +\sum_{k \in \mathcal{K}} \frac{\tilde{P}_{E M S}^{k}(1, b) \tilde{C}_{E M S}^{k 1}}{\tilde{V A} A^{\mathcal{K} 1}} \frac{\tilde{P}_{E M S}^{k}(1, b)-\tilde{P}_{E M S}^{k}(0, b)}{\tilde{P}_{E M S}^{k}(1, b)} \frac{\tilde{C}_{E M S}^{k 1}-\tilde{C}_{E M S}^{k 0}}{\tilde{C}_{E M S}^{k 1}}
\end{aligned}
$$


Notice that $\tilde{P}_{R}^{k}(1, b) \tilde{R}^{k 1} / \tilde{V A} A^{\mathcal{K} 1}=R^{k 1} / V A^{\mathcal{K} 1}$, which is the comparison period share of nominal revenue of unit $k$ in aggregate nominal value added, and that $\tilde{P}_{E M S}^{k}(1, b) \tilde{C}_{E M S}^{k 1} / \tilde{V A^{\mathcal{K} 1}}=C_{E M S}^{k 1} / V A^{\mathcal{K} 1}$, which is the comparison period share of nominal intermediate input cost of unit $k$ in aggregate nominal value added. The right-hand side of expression (13) consists of six terms: the first and the fourth give the aggregate effect of quantity change, the second and the fifth give the aggregate effect of relative price change, and the third and the sixth give the aggregate effect of the interaction of quantity and price change.

Forward- and backward-looking relative changes are related in a straightforward way. For instance,

$$
\frac{\tilde{R}^{k 1}-\tilde{R}^{k 0}}{\tilde{R}^{k 1}}=1-\left(1+\frac{\tilde{R}^{k 1}-\tilde{R}^{k 0}}{\tilde{R}^{k 0}}\right)^{-1} .
$$

The decompositions (9) and (12), (10) and (13) respectively, have the same structure, and it is by and large a matter of taste and convenience which of the two is preferred. Therefore, as another alternative the simple arithmetic mean of the Laspeyres- and Paasche-perspective decompositions (9) and (12) could be chosen. The resulting decomposition is named after Bennet and has the additional feature that the two interaction terms cancel:

$$
\begin{aligned}
& \tilde{V A^{\mathcal{K} 1}}-\tilde{V A^{\mathcal{K} 0}}= \\
& \sum_{k \in \mathcal{K}}(1 / 2)\left(\tilde{P}_{R}^{k}(1, b)+\tilde{P}_{R}^{k}(0, b)\right)\left(\tilde{R}^{k 1}-\tilde{R}^{k 0}\right) \\
& +\sum_{k \in \mathcal{K}}(1 / 2)\left(\tilde{P}_{R}^{k}(1, b)-\tilde{P}_{R}^{k}(0, b)\right)\left(\tilde{R}^{k 1}+\tilde{R}^{k 0}\right) \\
& -\sum_{k \in \mathcal{K}}(1 / 2)\left(\tilde{P}_{E M S}^{k}(1, b)+\tilde{P}_{E M S}^{k}(0, b)\right)\left(\tilde{C}_{E M S}^{k 1}-\tilde{C}_{E M S}^{k 0}\right) \\
& -\sum_{k \in \mathcal{K}}(1 / 2)\left(\tilde{P}_{E M S}^{k}(1, b)-\tilde{P}_{E M S}^{k}(0, b)\right)\left(\tilde{C}_{E M S}^{k 1}+\tilde{C}_{E M S}^{k 0}\right) .
\end{aligned}
$$

Switching to forward- or backward-looking relative changes would destroy the symmetry, as one easily checks. The symmetry can be retained when changes are related to the logarithmic mean, as $i^{2}$

\footnotetext{
${ }^{2}$ The logarithmic mean is, for any two strictly positive real numbers $a$ and $b$, defined by $L(a, b) \equiv(a-b) / \ln (a / b)$ if $a \neq b$ and $L(a, a) \equiv a$. It has the following properties: (1)
} 


$$
\frac{\tilde{V A}^{\mathcal{K} 1}-\tilde{V A^{\mathcal{K} 0}}}{L\left(\tilde{V A^{\mathcal{K} 1}}, \tilde{V A^{\mathcal{K} 0}}\right)}=\ln \left(\tilde{V A^{\mathcal{K}} 1} / \tilde{V A^{\mathcal{K} 0}}\right) .
$$

Inverting the logarithms then delivers

$$
\begin{aligned}
& \frac{\tilde{V A^{\mathcal{K} 1}}}{\tilde{V A^{\mathcal{K} 0}}}= \\
& \quad \exp \left\{\sum_{k \in \mathcal{K}} \frac{\left(\tilde{P}_{R}^{k}(1, b)+\tilde{P}_{R}^{k}(0, b)\right) L\left(\tilde{R}^{k 1}, \tilde{R}^{k 0}\right)}{2 L\left(\tilde{V A^{\mathcal{K} 1}}, \tilde{V A^{\mathcal{K} 0}}\right)} \ln \left(\frac{\tilde{R}^{k 1}}{\tilde{R}^{k 0}}\right)\right\} \\
& \quad \times \exp \left\{\sum_{k \in \mathcal{K}} \frac{L\left(\tilde{P}_{R}^{k}(1, b), \tilde{P}_{R}^{k}(0, b)\right)\left(\tilde{R}^{k 1}+\tilde{R}^{k 0}\right)}{2 L\left(\tilde{V A} A^{\mathcal{K} 1}, \tilde{V A} A^{\mathcal{K} 0}\right)} \ln \left(\frac{\tilde{P}_{R}^{k}(1, b)}{\tilde{P}_{R}^{k}(0, b)}\right)\right\} \\
& \quad \times \exp \left\{-\sum_{k \in \mathcal{K}} \frac{\left(\tilde{P}_{E M S}^{k}(1, b)+\tilde{P}_{E M S}^{k}(0, b)\right) L\left(\tilde{C}_{E M S}^{k 1}, \tilde{C}_{E M S}^{k 0}\right)}{2 L\left(\tilde{V A} A^{\mathcal{K} 1}, \tilde{V A} A^{\mathcal{K} 0}\right)} \ln \left(\frac{\tilde{C}_{E M S}^{k 1}}{\tilde{C}_{E M S}^{k 0}}\right)\right\} \\
& \quad \times \exp \left\{-\sum_{k \in \mathcal{K}} \frac{L\left(\tilde{P}_{E M S}^{k}(1, b), \tilde{P}_{E M S}^{k}(0, b)\right)\left(\tilde{C}_{E M S}^{k 1}+\tilde{C}_{E M S}^{k 0}\right)}{2 L\left(\tilde{V A} A^{\mathcal{K} 1}, \tilde{V A} A^{\mathcal{K} 0}\right)} \ln \left(\frac{\tilde{P}_{E M S}^{k}(1, b)}{\tilde{P}_{E M S}^{k}(0, b)}\right)\right\} .
\end{aligned}
$$

What do we see here? Aggregate real value added change, expressed as a ratio, is decomposed into four terms. Each term is a weighted product of subaggregate changes. The first term gives the total effect of real gross output quantity change, where each individual term is weighted by the share of mean revenue in mean aggregate value added. The second term gives the total effect of relative gross output price change, where each individual term is also weighted by the share of mean revenue in mean aggregate value added. The third term gives the total effect of real intermediate input quantity change, where each individual term is weighted by the share of mean intermediate input cost in mean aggregate value added. The fourth term gives the total effect of relative intermediate input price change, where each individual term is also weighted by the share of mean intermediate input cost in mean aggregate value added.

Though symmetric with respect to time, the weights in the first and second term, and those in the third and fourth term, are not equal, due $\overline{\min (a, b) \leq L(a, b) \leq \max (a, b) ;(2)} L(a, b)$ is continuous; (3) $L(\lambda a, \lambda b)=\lambda L(a, b)(\lambda>0)$; (4) $L(a, b)=L(b, a) ;(5)(a b)^{1 / 2} \leq L(a, b) \leq(a+b) / 2$; (6) $L(a, 1)$ is concave. See Balk (2008) for details. 
to different combinations of arithmetic and logarithmic means. This is not completely satisfactory. Another disadvantage of this decomposition is that aggregate real value added change is not written as a weighted product of subaggregate real value added changes.

\section{Symmetric decompositions for aggregate out- put change}

When change is measured as ratio the following symmetric decomposition can be used (see Balk 2003 and 2009):

$$
\frac{V A^{\mathcal{K} 1}}{V A^{\mathcal{K} 0}}=\exp \left\{\sum_{k \in \mathcal{K}} \Psi^{k} \ln \left(\frac{V A^{k 1}}{V A^{k 0}}\right)\right\}
$$

where

$$
\Psi^{k} \equiv \frac{L\left(\frac{V A^{k 1}}{V A^{\mathcal{1} 1}}, \frac{V A^{k 0}}{V A^{\mathcal{K} 0}}\right)}{\sum_{k \in \mathcal{K}} L\left(\frac{V A^{k 1}}{V A^{\mathcal{K} 1}}, \frac{V A^{k 0}}{V A^{\mathcal{K} 0}}\right)}(k \in \mathcal{K})
$$

Aggregate nominal value-added change, measured as ratio, is thus equal to a weighted geometric mean of individual nominal value-added changes. Each coefficient $\Psi^{k}$ is the (normalized) mean share of production unit $k$ in aggregate nominal value added. Notice that these coefficients add up to 1 ; that is,

$$
\sum_{k \in \mathcal{K}} \Psi^{k}=1
$$

Next, each individual nominal value-added change can be decomposed as (see Balk 2010a or 2010b, Appendix B)

$$
\frac{V A^{k 1}}{V A^{k 0}}=\frac{P_{R}^{k}(1,0)^{\phi_{R}^{k}}}{P_{E M S}^{k}(1,0)^{\phi_{E M S}^{k}}} \frac{Q_{R}^{k}(1,0)^{\phi_{R}^{k}}}{Q_{E M S}^{k}(1,0)^{\phi_{E M S}^{k}}}(k \in \mathcal{K}),
$$

where the exponent $\phi_{R}^{k}$ is defined as $\phi_{R}^{k} \equiv L\left(R^{k 1}, R^{k 0}\right) / L\left(V A^{k 1}, V A^{k 0}\right)$; that is, mean nominal revenue over mean nominal value added; and the exponent $\phi_{E M S}^{k}$ is defined as $\phi_{E M S}^{k} \equiv L\left(C_{E M S}^{k 1}, C_{E M S}^{k 0}\right) / L\left(V A^{k 1}, V A^{k 0}\right)$; that is, mean nominal intermediate inputs cost over mean nominal value added. It is here presupposed that the price and quantity indices for revenue and intermediate 
inputs cost satisfy product relations (5) and (6). Notice that $\phi_{R}^{k}-\phi_{E M S}^{k} \leq 1$ $(k \in \mathcal{K})$, due to the concavity of the logarithmic mean.

When we define the unit $k$ price index and quantity index of value added respectively as

$$
\begin{aligned}
P_{V A}^{k}(1,0) & \equiv \frac{P_{R}^{k}(1,0)^{\phi_{R}^{k}}}{P_{E M S}^{k}(1,0)^{\phi_{E M S}^{k}}}(k \in \mathcal{K}) \\
Q_{V A}^{k}(1,0) & \equiv \frac{Q_{R}^{k}(1,0)^{\phi_{R}^{k}}}{Q_{E M S}^{k}(1,0)^{\phi_{E M S}^{k}}}(k \in \mathcal{K}),
\end{aligned}
$$

then expression (20) can be simplified to

$$
\frac{V A^{k 1}}{V A^{k 0}}=P_{V A}^{k}(1,0) Q_{V A}^{k}(1,0)(k \in \mathcal{K}) .
$$

Technically seen, these value added price and quantity indices are (generalized) Montgomery-Vartia indices.

Substituting expression (20) into (18) delivers an expression,

$$
\frac{V A^{\mathcal{K} 1}}{V A^{\mathcal{K} 0}}=\prod_{k \in \mathcal{K}}\left(\frac{P_{R}^{k}(1,0)^{\phi_{R}^{k} \Psi^{k}}}{P_{E M S}^{k}(1,0)^{\phi_{E M S}^{k} \Psi^{k}}}\right) \prod_{k \in \mathcal{K}}\left(\frac{Q_{R}^{k}(1,0)^{\phi_{R}^{k} \Psi^{k}}}{Q_{E M S}^{k}(1,0)^{\phi_{E M S}^{k} \Psi^{k}}}\right),
$$

which is perfectly symmetric. Notice that the exponent $\phi_{R}^{k} \Psi^{k}$ can be interpreted as the share of nominal revenue of production unit $k$ in aggregate nominal value added; or, as a so-called Domar weight. These weights, however, do not add up to 1 . Similarly, the exponent $\phi_{E M S}^{k} \Psi^{k}$ can be interpreted as the share of nominal intermediate inputs cost of unit $k$ in aggregate nominal value added. These weights also do not add up to 1 .

Aggregate output change can now be defined as the quantity component of $V A^{\mathcal{K} 1} / V A^{\mathcal{K} 0}$; hence as

$$
Q_{V A}^{\mathcal{K}}(1,0) \equiv \prod_{k \in \mathcal{K}}\left(\frac{Q_{R}^{k}(1,0)^{\phi_{R}^{k} \Psi^{k}}}{Q_{E M S}^{k}(1,0)^{\phi_{E M S}^{k} \Psi^{k}}}\right)=\frac{\prod_{k \in \mathcal{K}} Q_{R}^{k}(1,0)^{\phi_{R}^{k} \Psi^{k}}}{\prod_{k \in \mathcal{K}} Q_{E M S}^{k}(1,0)^{\phi_{E M S}^{k} \Psi^{k}}}
$$

The right-hand side of this expression enables us to decompose aggregate output change according to various viewpoints: the contribution of gross output and intermediate inputs, the contribution of the production units making up the ensemble, or a combination of these. 
Definition (25) is, however, not unique. As an alternative to expression (24) consider

$$
\frac{V A^{\mathcal{K} 1}}{V A^{\mathcal{K} 0}}=\prod_{k \in \mathcal{K}}\left(\frac{P_{R}^{k}(1,0)^{\xi_{R}^{k}}}{P_{E M S}^{k}(1,0)^{\xi_{E M S}^{k}}}\right) \prod_{k \in \mathcal{K}}\left(\frac{Q_{R}^{k}(1,0)^{\xi_{R}^{k}}}{Q_{E M S}^{k}(1,0)^{\xi_{E M S}^{k}}}\right),
$$

where the exponent $\xi_{R}^{k}$ is defined as

$$
\xi_{R}^{k} \equiv \frac{L\left(\frac{R^{k 1}}{V A^{\mathcal{K} 1}}, \frac{R^{k 0}}{V A^{\mathcal{K} 0}}\right)}{\sum_{k \in \mathcal{K}} L\left(\frac{R^{k 1}}{V A^{\mathcal{K} 1}}, \frac{R^{k 0}}{V A^{\mathcal{K} 0}}\right)-\sum_{k \in \mathcal{K}} L\left(\frac{C_{E M S}^{k 1}}{V A^{\mathcal{K} 1}}, \frac{C_{E M S}^{k 0}}{V A^{\mathcal{K} 0}}\right)}(k \in \mathcal{K}),
$$

and the exponent $\xi_{E M S}^{k}$ as

$$
\xi_{E M S}^{k} \equiv \frac{L\left(\frac{C_{E M S}^{k 1}}{V A^{\mathcal{K} 1}}, \frac{C_{E M S}^{k 0}}{V A^{\mathcal{K} 0}}\right)}{\sum_{k \in \mathcal{K}} L\left(\frac{R^{k 1}}{V A^{\mathcal{C} 1}}, \frac{R^{k 0}}{V A^{\mathcal{K} 0}}\right)-\sum_{k \in \mathcal{K}} L\left(\frac{C_{E M S}^{k 1}}{V A^{\mathcal{L} 1}}, \frac{C_{E M S}^{k 0}}{V A^{\mathcal{K} 0}}\right)}(k \in \mathcal{K}) .
$$

For the derivation of expression (26) the reader is referred to the Appendix. ${ }^{3}$ The exponent $\xi_{R}^{k}$ can be interpreted as the (normalized) mean share of nominal revenue of production unit $k$ in aggregate nominal value added; or, again as a Domar weight. Similarly, the exponent $\xi_{E M S}^{k}$ can be interpreted as the (normalized) mean share of nominal intermediate inputs cost of unit $k$ in aggregate nominal value added. The difference of these exponents adds up to 1 ; that is, $\sum_{k \in \mathcal{K}} \xi_{R}^{k}-\sum_{k \in \mathcal{K}} \xi_{E M S}^{k}=1$.

Based on expression (26), the quantity component of $V A^{\mathcal{K} 1} / V A^{\mathcal{K} 0}$, defining aggregate output change, is given by

$$
Q_{V A}^{\mathcal{K}}(1,0) \equiv \prod_{k \in \mathcal{K}}\left(\frac{Q_{R}^{k}(1,0)^{\xi_{R}^{k}}}{Q_{E M S}^{k}(1,0)^{\xi_{E M S}^{k}}}\right)=\frac{\prod_{k \in \mathcal{K}} Q_{R}^{k}(1,0)^{\xi_{R}^{k}}}{\prod_{k \in \mathcal{K}} Q_{E M S}^{k}(1,0)^{\xi_{E M S}^{k}}} .
$$

Again, the right-hand side of this expression enables us to decompose aggregate output change according to various viewpoints: the contribution of gross output and intermediate inputs, the contribution of the production units making up the ensemble, or a combination of these. Notice that, though expressions (25) and (27) have the same structure, the exponents are different:

\footnotetext{
${ }^{3}$ Reinsdorf and Yuskavage (2010) developed a similar formula in a slightly different context.
} 
$\xi_{R}^{k} \neq \phi_{R}^{k} \Psi^{k}$ and $\xi_{E M S}^{k} \neq \phi_{E M S}^{k} \Psi^{k}$. The influence of these differences on the outcome will in general be immaterial.

The reason is that difference between expressions (25) and (27) is that between a one-step and a two-step procedure. Expression (27) is the result of a one-step procedure: nominal revenue (or intermediate inputs cost) of unit $k$ is immediately related to aggregate nominal value added. Expression (25) is the result of a two-step procedure: nominal revenue (or intermediate inputs cost) of unit $k$ is first related to nominal value added of unit $k$, and then nominal value added of unit $k$ is related to aggregate nominal value added.

\section{$5 \quad$ Labour productivity}

Let $L^{k t}$ be a scalar measure of the quantity of labour used by production unit $k$ during period $t$ (such as hours of work or full-time-equivalent jobs), and let $L^{\mathcal{K} t} \equiv \sum_{k \in \mathcal{K}} L^{k t}$. Following conventional practice, DTW define aggregate labour productivity as aggregate real value added divided by the total quantity of labour; that is, $\widetilde{V A^{\mathcal{K} t}} / L^{\mathcal{K} t}$. Then, using expression (7), it appears that aggregate labour productivity can be written as

$$
\tilde{V A}^{\mathcal{K} t} / L^{\mathcal{K} t}=\sum_{k \in \mathcal{K}}\left(s_{R}^{k t} \tilde{R}^{k t} / L^{k t}-s_{E M S}^{k t} \tilde{C}_{E M S}^{k t} / L^{k t}\right)
$$

where $s_{R}^{k t} \equiv\left(L^{k t} / L^{\mathcal{K} t}\right) \tilde{P}_{R}^{k}(t, b)$ is the labour input share of unit $k$ adjusted by the relative gross output price index, and $s_{E M S}^{k t} \equiv\left(L^{k t} / L^{\mathcal{K} t}\right) \tilde{P}_{E M S}^{k}(t, b)$ is the same labour input share but now adjusted by the relative intermediate inputs price index. Notice that $\tilde{R}^{k t} / L^{k t}$ is real gross output per unit of labour, whereas $\tilde{C}_{E M S}^{k t} / L^{k t}$ is real intermediate inputs use per unit of labour.

Next, DTW define labour productivity growth as

$$
\frac{\tilde{V A^{\mathcal{K} 1}} / L^{\mathcal{K} 1}-\tilde{V A^{\mathcal{K} 0}} / L^{\mathcal{K} 0}}{\tilde{V A} A^{\mathcal{K} 0} / L^{\mathcal{K} 0}}
$$

and decompose this according to the Laspeyres, forward-looking perspective, as in Section 2. This delivers an expression providing 2 times 3 effects: change of a relative price index adjusted labour input share, quantity-perunit-of-labour change, and their interaction. 
Again, instead of the Laspeyres-perspective one could use the Paasche, backward-looking perspective, or the symmetric Bennet decomposition. The disadvantage of all these decompositions is that they do not provide an expression for aggregate labour productivity change as a weighted sum or product of labour productivity changes at the individual production unit level. Another disadvantage of using expression (28) as point of departure is that this only works when labour input can be measured by a single, scalar variable. When labour input is more complex, which in reality almost always is the case, so that indexes instead of scalars must be used, expression (28) appears to be difficult to generalize. Thus, an other approach is called for.

This approach starts from considering nominal value added per unit of labour and the development thereof. Applying expression (18) delivers

$$
\begin{aligned}
\ln \left(\frac{V A^{\mathcal{K} 1} / L^{\mathcal{K} 1}}{V A^{\mathcal{K} 0} / L^{\mathcal{K} 0}}\right) & =\ln \left(\frac{V A^{\mathcal{K} 1}}{V A^{\mathcal{K} 0}}\right)-\ln \left(\frac{L^{\mathcal{K} 1}}{L^{\mathcal{K} 0}}\right) \\
& =\sum_{k \in \mathcal{K}} \Psi^{k} \ln \left(\frac{V A^{k 1}}{V A^{k 0}}\right)-\sum_{k \in \mathcal{K}} \xi_{L}^{k} \ln \left(\frac{L^{k 1}}{L^{k 0}}\right)
\end{aligned}
$$

where $\Psi^{k}$ was defined earlier and $\xi_{L}^{k}$ is defined analogously as

$$
\xi_{L}^{k} \equiv \frac{L\left(\frac{L^{k 1}}{L^{\mathcal{K} 1}}, \frac{L^{k 0}}{L^{\mathcal{K} 0}}\right)}{\sum_{k \in \mathcal{K}} L\left(\frac{L^{k 1}}{L^{\mathcal{K} 1}}, \frac{L^{k 0}}{L^{K 0}}\right)}(k \in \mathcal{K}) .
$$

Notice that

$$
\sum_{k \in \mathcal{K}} \xi_{L}^{k}=1
$$

Next, use expression (23) to obtain

$$
\begin{aligned}
& \ln \left(\frac{V A^{\mathcal{K} 1} / L^{\mathcal{K} 1}}{V A^{\mathcal{K} 0} / L^{\mathcal{K} 0}}\right)= \\
& \quad \sum_{k \in \mathcal{K}} \Psi^{k} \ln P_{V A}^{k}(1,0)+\sum_{k \in \mathcal{K}} \Psi^{k} \ln Q_{V A}^{k}(1,0)-\sum_{k \in \mathcal{K}} \xi_{L}^{k} \ln \left(\frac{L^{k 1}}{L^{k 0}}\right) .
\end{aligned}
$$


The first term at the right-hand side of this expression measures the price component of the change of value-added-per-unit-of-labour. Thus the remaining two components together measure aggregate labour productivity change. Hence, the index of aggregate labour productivity can be defined as

$$
\ln I L P R O D^{\mathcal{K}}(1,0) \equiv \sum_{k \in \mathcal{K}} \Psi^{k} \ln Q_{V A}^{k}(1,0)-\sum_{k \in \mathcal{K}} \xi_{L}^{k} \ln \left(\frac{L^{k 1}}{L^{k 0}}\right)
$$

When unit k's (value-added based) labour productivity index is, quite naturally, defined as

$$
\operatorname{ILPROD} D^{k}(1,0) \equiv \frac{Q_{V A}^{k}(1,0)}{L^{k 1} / L^{k 0}}(k \in \mathcal{K}),
$$

then expression (32) can be rearranged in the following, analytically useful, form:

$$
\begin{aligned}
& \ln I L P R O D^{\mathcal{K}}(1,0)= \\
& \quad \sum_{k \in \mathcal{K}} \Psi^{k} \ln I L P R O D^{k}(1,0)+\sum_{k \in \mathcal{K}}\left(\Psi^{k}-\xi_{L}^{k}\right)\left(\ln \left(\frac{L^{k 1}}{L^{k 0}}\right)-a\right),
\end{aligned}
$$

where $a$ is an arbitrary scalar. Recall that $\sum_{k \in \mathcal{K}}\left(\Psi^{k}-\xi_{L}^{k}\right)=0$. Expression (34) decomposes aggregate labour productivity change into two components, the first of which is a weighted mean of all the unit-specific labour productivity changes. The weights are the shares of the units in aggregate value added. Setting $a$ equal to the unweighted mean of $\ln \left(L^{k 1} / L^{k 0}\right)$ a nice interpretation of the remainder term emerges, namely as the covariance of labour quantity change and the gap between a unit's value added share and its labour share. This covariance is positive if a higher-than-average (lower-than-average) increase of the quantity of labour corresponds with a relatively big (small) gap between the unit's share in value added and its labour share, the average gap being equal to zero.

Due to the fact that the coefficients $\Psi^{k}$ and $\xi_{L}^{k}$ add up to 1 , the remainder term can also be written as

$$
\sum_{k \in \mathcal{K}}\left(\Psi^{k}-\xi_{L}^{k}\right)\left(\ln \left(\frac{L^{k 1}}{L^{k 0}}\right)-a\right)=\sum_{k \in \mathcal{K}} \Psi^{k} \ln \left(\frac{L^{k 1} / L^{\mathcal{K} 1}}{L^{k 0} / L^{\mathcal{K} 0}}\right) .
$$


This is a weighted mean of labour share ratios. Thus the covariance term in expression (34) can be interpreted as the effect of labour reallocation on aggregate labour productivity change. Expression (34) is the discrete-time version of equation (7) of Stiroh (2002) and corresponds to equation (1) of Nordhaus (2002).

The following alternative rearrangement of expression (32) is also worthwile to consider:

$$
\begin{aligned}
& \ln I L P R O D^{\mathcal{K}}(1,0)= \\
& \quad \sum_{k \in \mathcal{K}} \xi_{L}^{k} \ln I L P R O D^{k}(1,0)+\sum_{k \in \mathcal{K}}\left(\Psi^{k}-\xi_{L}^{k}\right)\left(\ln Q_{V A}^{k}(1,0)-b\right),
\end{aligned}
$$

where $b$ is an arbitrary scalar. The first component of expression (36) is again a weighted mean of unit-specific labour productivity changes, the weights now being equal to the labour shares. Setting $b$ equal to the unweighted mean of $\ln Q_{V A}^{k}(1,0)$ the remainder term can be interpreted as the covariance of valueadded quantity change and the gap between a unit's value added share and its labour share. This covariance is positive if a higher-than-average (lowerthan-average) increase of the quantity component of value added corresponds with a relatively big (small) gap between the unit's share in value added and its labour share. Expressions (34) and (36) correspond to expression (20') of Reinsdorf and Yuskavage (2010). ${ }^{4}$

A third alternative emerges by substituting the definition of $\ln Q_{V A}^{k}(1,0)$ back into expression (32). This delivers

$$
\begin{aligned}
& \ln I L P R O D^{\mathcal{K}}(1,0)= \\
& \sum_{k \in \mathcal{K}} \Psi^{k} \phi_{R}^{k} \ln Q_{R}^{k}(1,0)-\sum_{k \in \mathcal{K}} \Psi^{k} \phi_{E M S}^{k} \ln Q_{E M S}^{k}(1,0)-\sum_{k \in \mathcal{K}} \xi_{L}^{k} \ln \left(\frac{L^{k 1}}{L^{k 0}}\right)= \\
& \sum_{k \in \mathcal{K}} \Psi^{k} \phi_{R}^{k} \ln \left(\frac{Q_{R}^{k}(1,0)}{L^{k 1} / L^{k 0}}\right)-\sum_{k \in \mathcal{K}} \Psi^{k} \phi_{E M S}^{k} \ln \left(\frac{Q_{E M S}^{k}(1,0)}{L^{k 1} / L^{k 0}}\right)+
\end{aligned}
$$

\footnotetext{
${ }^{4}$ Instead of nominal value added, Reinsdorf and Yuskavage (2010) started by considering value added expressed in artificial prices, defined as a weighted mean of period 0 and period 1 prices. The left-hand side of expression (31) can then be written as $\ln \left(Q_{V A}^{\mathcal{K}}(1,0) /\left(L^{\mathcal{K} 1} / L^{\mathcal{K} 0}\right)\right)$, where $Q_{V A}^{\mathcal{K}}(1,0)$ is a value-added based aggregate output quantity index. The first term at the right-hand side of the said expression however not necessarily disappears.
} 


$$
\sum_{k \in \mathcal{K}}\left(\Psi^{k} \phi_{R}^{k}-\Psi^{k} \phi_{E M S}^{k}-\xi_{L}^{k}\right)\left(\ln \left(\frac{L^{k 1}}{L^{k 0}}\right)\right) .
$$

The first term at the right-hand side is a weighted sum of unit-specific grossoutput based labour productivity indices, the weights being $\Psi^{k} \phi_{R}^{k}$, i.e. Domar weights. The second term is a weighted sum of unit-specific indices of intermediate inputs deepening, the weights being the counterparts of the Domar weights, i.e. $\Psi^{k} \phi_{E M S}^{k}$. Since $\phi_{R}^{k}-\phi_{E M S}^{k} \leq 1$, the coefficients in the third term $\Psi^{k} \phi_{R}^{k}-\Psi^{k} \phi_{E M S}^{k}-\xi_{L}^{k} \leq \Psi^{k}-\xi_{L}^{k}$, and the discrepancy should usually be small. Thus the last term of expression (37) has the same structure as the last term of expression (34). Expression (37) was favored by Karagiannis (2011).

Expression (32) is, however, not unique. Instead of expression (20) one could use expression (26) to obtain

$$
\begin{aligned}
& \ln \left(\frac{V A^{\mathcal{K} 1} / L^{\mathcal{K} 1}}{V A^{\mathcal{K} 0} / L^{\mathcal{K} 0}}\right)= \\
& \quad \sum_{k \in \mathcal{K}} \ln \left(\frac{P_{R}^{k}(1,0)^{\xi_{R}^{k}}}{P_{E M S}^{k}(1,0)^{\xi_{E M S}^{k}}}\right)+\sum_{k \in \mathcal{K}} \ln \left(\frac{Q_{R}^{k}(1,0)^{\xi_{R}^{k}}}{Q_{E M S}^{k}(1,0)^{\xi_{E M S}^{k}}}\right) \\
& \quad-\sum_{k \in \mathcal{K}} \xi_{L}^{k} \ln \left(\frac{L^{k 1}}{L^{k 0}}\right) .
\end{aligned}
$$

In this case aggregate labour productivity change is defined as

$$
\begin{aligned}
& \ln I L P R O D^{\mathcal{K}}(1,0) \equiv \\
& \sum_{k \in \mathcal{K}} \xi_{R}^{k} \ln Q_{R}^{k}(1,0)-\sum_{k \in \mathcal{K}} \xi_{E M S}^{k} \ln Q_{E M S}^{k}(1,0)-\sum_{k \in \mathcal{K}} \xi_{L}^{k} \ln \left(\frac{L^{k 1}}{L^{k 0}}\right) .
\end{aligned}
$$

This expression has the same structure as the middle part of expression (37), the weights in the first two terms being slightly different. Expression (39) can be rearranged to

$$
\begin{aligned}
& \ln I L P R O D^{\mathcal{K}}(1,0)= \\
& \qquad \sum_{k \in \mathcal{K}}\left(\xi_{R}^{k}-\xi_{E M S}^{k}\right) \ln \left(\frac{Q_{R}^{k}(1,0)}{L^{k 1} / L^{k 0}}\right)+\sum_{k \in \mathcal{K}} \xi_{E M S}^{k} \ln \left(\frac{Q_{R}^{k}(1,0)}{Q_{E M S}^{k}(1,0)}\right)
\end{aligned}
$$




$$
+\sum_{k \in \mathcal{K}}\left(\xi_{R}^{k}-\xi_{E M S}^{k}-\xi_{L}^{k}\right)\left(\ln \left(\frac{L^{k 1}}{L^{k 0}}\right)-a\right) .
$$

Notice that $\sum_{k \in \mathcal{K}}\left(\xi_{R}^{k}-\xi_{E M S}^{k}-\xi_{L}^{k}\right)=0$. The first component at the righthand side of expression (40) is a weighted sum of all the unit-specific grossoutput based labour productivity changes, the weights being (approximately equal to) the value-added shares of the units. The second component is the aggregate excess of gross output quantity change over intermediate inputs quantity change, the weights being the intermediate inputs cost shares in aggregate value added. The third component expresses again the labour reallocation effect. The entire expression (40) corresponds to expression (20) of Reinsdorf and Yuskavage (2004) and is the discrete-time version of expression (6) of Stiroh (2002). The said expression was also used by Bosworth and Triplett (2007).

\section{Conclusion}

When dealing with an ensemble of production units it is important to relate aggregate output change to output change at the level of the production units. An important measure of output change is real value added change. In this paper we considered symmetric decompositions of aggregate real value added change. Two, slightly different, solutions are given by expressions (25) and (27).

We proceeded by considering labour productivity change. The solutions are here basically given by expressions (32) and (39). Additional insight into the "causes" of aggregate labour productivity change can be obtained from expression such as (34), (36), (37) and (40).

Three of these expressions contain a labour reallocation term, also called "Denison effect". As Bosworth and Triplett (2007) observe, the interpretation of such a term is not immediately intuitive. Their comment runs as follows:

"Consider a technological shock in industry A that raises (...) labour productivity, and for the sake of the illustration we specify that technologies in other industries are unchanged. Unless the demand elasticity for industry A's output is high, industry A will use fewer resources. If the released resources go to industries 
with lower productivity growth rates, the reallocation reduces aggregate and sector productivity rates (the direct rates). Reallocations thus provide a partial offset to the direct impact on the sector rates from industry A's productivity gain."

Mathematically seen, the labour reallocation effect occurs to the extent that a unit's value added share $\Psi^{k}$ or $\xi_{R}^{k}-\xi_{E M S}^{k}$ differs from its labour share $\xi_{L}^{k}$. If all those shares would stay equal, there would be no reallocation effect.

Finally, in all the labour productivity expressions labour quantities figure as scalars. When labour is more complex, so that indices rather than scalars must be used, these expressions can easily be generalized. All one has to do is replace each ratio $L^{k 1} / L^{k 0}$ by a quantity index $Q_{L}^{k}(1,0)$, and each weight $\xi_{L}^{k}$ by production unit $k$ 's share in aggregate labour cost. 


\section{Appendix: Proof of expression (26)}

Point of departure is the identity

$$
\sum_{k \in \mathcal{K}} \frac{V A^{k 1}}{V A^{\mathcal{K} 1}}-\sum_{k \in \mathcal{K}} \frac{V A^{k 0}}{V A^{\mathcal{K} 0}}=0
$$

Using the definition of value added, this can be rewritten as

$$
\sum_{k \in \mathcal{K}} \frac{R^{k 1}-C_{E M S}^{k 1}}{V A^{\mathcal{K} 1}}-\sum_{k \in \mathcal{K}} \frac{R^{k 0}-C_{E M S}^{k 0}}{V A^{\mathcal{K} 0}}=0,
$$

which can be rearranged to

$$
\sum_{k \in \mathcal{K}}\left(\frac{R^{k 1}}{V A^{\mathcal{K} 1}}-\frac{R^{k 0}}{V A^{\mathcal{K} 0}}\right)-\sum_{k \in \mathcal{K}}\left(\frac{C_{E M S}^{k 1}}{V A^{\mathcal{K} 1}}-\frac{C_{E M S}^{k 0}}{V A^{\mathcal{K} 0}}\right)=0,
$$

Applying the definition of the logarithmic mean, the last expression can be rewritten as

$$
\begin{aligned}
\sum_{k \in \mathcal{K}} L & \left(\frac{R^{k 1}}{V A^{\mathcal{K} 1}}, \frac{R^{k 0}}{V A^{\mathcal{K} 0}}\right) \ln \left(\frac{R^{k 1} / V A^{\mathcal{K} 1}}{R^{k 0} / V A^{\mathcal{K} 0}}\right) \\
& -\sum_{k \in \mathcal{K}} L\left(\frac{C_{E M S}^{k 1}}{V A^{\mathcal{K} 1}}, \frac{C_{E M S}^{k 0}}{V A^{\mathcal{K} 0}}\right) \ln \left(\frac{C_{E M S}^{k 1} / V A^{\mathcal{K} 1}}{C_{E M S}^{k 0} / V A^{\mathcal{K} 0}}\right)=0 .
\end{aligned}
$$

This, finally, can be rearranged as

$$
\ln \left(\frac{V A^{\mathcal{K} 1}}{V A^{\mathcal{K} 0}}\right)=\sum_{k \in \mathcal{K}} \xi_{R}^{k} \ln \left(\frac{R^{k 1}}{R^{k 0}}\right)-\sum_{k \in \mathcal{K}} \xi_{E M S}^{k} \ln \left(\frac{C_{E M S}^{k 1}}{C_{E M S}^{k 0}}\right),
$$

where

$$
\xi_{R}^{k} \equiv \frac{L\left(\frac{R^{k 1}}{V A^{\mathcal{K} 1}}, \frac{R^{k 0}}{V A^{\mathcal{K} 0}}\right)}{\sum_{k \in \mathcal{K}} L\left(\frac{R^{k 1}}{V A^{\mathcal{K} 1}}, \frac{R^{k 0}}{V A^{\mathcal{K} 0}}\right)-\sum_{k \in \mathcal{K}} L\left(\frac{C_{E M S}^{k 1}}{V A^{\mathcal{K} 1}}, \frac{C_{E M S}^{k 0}}{V A^{\mathcal{K} 0}}\right)}(k \in \mathcal{K}),
$$

and

$$
\xi_{E M S}^{k} \equiv \frac{L\left(\frac{C_{E M S}^{k 1}}{V A^{\mathcal{K} 1}}, \frac{C_{E M S}^{k 0}}{V A^{\mathcal{K} 0}}\right)}{\sum_{k \in \mathcal{K}} L\left(\frac{R^{k 1}}{V A^{\mathcal{K} 1}}, \frac{R^{k 0}}{V A^{\mathcal{K} 0}}\right)-\sum_{k \in \mathcal{K}} L\left(\frac{C_{E M S}^{k 1}}{V A^{\mathcal{K} 1}}, \frac{C_{E M S}^{k 0}}{V A^{\mathcal{K} 0}}\right)}(k \in \mathcal{K}) .
$$


Replacing revenue and intermediate inputs cost ratios by products of price and quantity indices, one obtains

$$
\begin{aligned}
& \ln \left(\frac{V A^{\mathcal{K} 1}}{V A^{\mathcal{K} 0}}\right)= \\
& \quad \sum_{k \in \mathcal{K}} \xi_{R}^{k} \ln \left(P_{R}^{k}(1,0) Q_{R}^{k}(1,0)\right)-\sum_{k \in \mathcal{K}} \xi_{E M S}^{k} \ln \left(P_{E M S}^{k}(1,0) Q_{E M S}^{k}(1,0)\right),
\end{aligned}
$$

from which expression (26) immediately follows. 


\section{References}

[1] Balk, B. M., 2003, "Ideal Indices and Indicators for Two or More Factors", Journal of Economic and Social Measurement 28, 203-217.

[2] Balk, B. M., 2008, Price and Quantity Index Numbers: Models for Measuring Aggregate Change and Difference (Cambridge University Press, New York).

[3] Balk, B. M., 2009, "Measuring and Relating Aggregate and Subaggregate Productivity Change without Neoclassical Assumptions", Discussion Paper 09026 (Statistics Netherlands, www.cbs.nl).

[4] Balk, B. M., 2010a, "Measuring Productivity Change without Neoclassical Assumptions: A Conceptual Analysis", in: Price and Productivity Measurement: Volume 6 - Index Number Theory, edited by W.E. Diewert, B.M. Balk, D. Fixler, K.J. Fox and A.O. Nakamura (Trafford Press, www.vancouvervolumes.com and www.indexmeasures.com).

[5] Balk, B. M., 2010b, "An Assumption-free Framework for Measuring Productivity Change", The Review of Income and Wealth 56, Special Issue 1, S224-S256.

[6] Bosworth, B. P. and J. E. Triplett, 2007, "The Early 21st Century U. S. Productivity Expansion is Still in Services", International Productivity Monitor 14, 3-19.

[7] Dufour, A., J. Tang and W. Wang, 2006, "Unbalanced Industry Demand and Supply Shifts: Implications for Economic Growth in Canada and the United States", Working Paper 2006-04 (Industry Canada, Ottawa). Presented at the World Congress on National Accounts and Economic Performance Measures for Nations, Arlington VA, 13-17 May 2008.

[8] Karagiannis, G., 2011, "Another Decomposition of Aggregate Labor Productivity Growth", Mimeo September 2011 (Department of Economics, University of Macedonia, Thessaloniki, Greece). Presented at the EWEPA, Verona, 21-24 June 2011.

[9] Nordhaus, W. D., 2002, "Productivity Growth and the New Economy", Brookings Papers on Economic Activity 2002(2), 211-244. 
[10] Reinsdorf, M. and R. Yuskavage, 2010, "Exact Industry Contributions to Labor Productivity Change", in: Price and Productivity Measurement: Volume 6 - Index Number Theory, edited by W.E. Diewert, B.M. Balk, D. Fixler, K.J. Fox and A.O. Nakamura (Trafford Press, www.vancouvervolumes.com and www.indexmeasures.com).

[11] Stiroh, K. J., 2002, "Information Technology and the U.S. Productivity Revival: What Do the Industry Data Say?", The American Economic Review 92, 1559-1576. 


\section{Publications in the Report Series Research* in Management}

\section{ERIM Research Program: "Marketing"}

2011

Nonmetric Unfolding of Marketing Data: Degeneracy and Stability

Michel van de Velden, Alain De Beuckelaer, Patrick J.F. Groenen, and Frank M.T.A. Busing

ERS-2011-006-MKT

http://hdl.handle.net/1765/22725

The Bright Side and Dark Side of Embedded Ties In Business-to-Business Innovation

Corine S. Noordhoff, Kyriakos Kyriakopoulos, Christine Moorman, Pieter Pauwels, and Benedict G.C. Dellaert ERS-2011-008-MKT

http://hdl.handle.net/1765/22813

The Global Entry of New Pharmaceuticals: A Joint Investigation of Launch Window and Price

Isabel Verniers, Stefan Stremersch, and Christophe Croux

ERS-2011-010-MKT

http://hdl.handle.net/1765/23488

Visualizing attitudes towards service levels

Kar Yin Lam, Michel Van de Velden, and Philip Hans Franses

ERS-2011-022-MKT

http://hdl.handle.net/1765/26471

Dissecting Aggregate Output and Labour Productivity Change

Bert M. Balk

ERS-2011-023-MKT

http://hdl.handle.net/1765/26893

* A complete overview of the ERIM Report Series Research in Management: https://ep.eur.nl/handle/1765/1

ERIM Research Programs:

LIS Business Processes, Logistics and Information Systems

ORG Organizing for Performance

MKT Marketing

F\&A Finance and Accounting

STR Strategy and Entrepreneurship 